\title{
Las nuevas competencias TIC en el personal de los servicios de salud
}

\begin{tabular}{c}
\hline Palabras clave \\
\hline competencias TIC \\
salud \\
internet
\end{tabular}

internet

\author{
Raúl Choque Larrauri \\ (Groningen, Holanda) \\ Universidad de Groningen
}

\begin{abstract}
Resumen
En el campo de la salud, con la incursión de las nuevas tecnologías de la información y la comunicación, se hace necesario el desarrollo de nuevas competencias en el uso de las TIC por parte del personal de los servicios de salud. Los médicos, enfermeros y otros profesionales están en permanente interacción con los pacientes. Utilizando las TIC, especialmente internet, se puede mejorar los procesos de comunicación brindándoles información oportuna sobre la prevención de enfermedades, la promoción de la salud, así como sobre el tratamiento de una enfermedad. Las nuevas competencias TIC habilitan una mayor interacción entre el personal de salud y los pacientes a través de los emails, SMS, chat, foros, páginas web, Facebook, Twitter y otros medios. Las principales competencias TIC son el acceso a información científica de salud, la gestión de la información y el conocimiento en salud, la generación de conocimiento en salud y la divulgación de conocimiento en salud. Las nuevas competencias TIC requieren que las personas sean alfabetizadas digitales, y son fundamentales para poder desenvolverse en este nuevo contexto socio tecnológico de la Sociedad Red en la cual nos encontramos viviendo.
\end{abstract}

Agradecimientos a Network on Humanitarian Assistance (NOHA) y al Departamento de Relaciones Internacionales de la Facultad de Artes de la Universidad de Groningen en Holanda, por permitirme realizar mis estudios post doctorales en el marco de Erasmus Mundus External Cooperation Windows - ARBOPEUE. Especiales agradecimientos al Dr. Joost Herman, Dr. Hub Hermans, Dra. Nina Hansen, Dr. Peter Tellegen, Mg. Renée Bakker y c.PhD Cecile de Milliano. En Perú mis agradecimientos a Cecilia Israel del Programa de Becas de la Fundación Ford. Contacto para correspondencia: Raul Choque Larrauri. Email:raul.choque@pucp.edu.pe. Teléfono: 0051-9878-20905. Twitter: @raulchoque 


\title{
New Competences in Information and Communication Technologies of Health Providers
}

\begin{tabular}{c}
\hline Keywords \\
\hline ICT skills \\
health \\
internet
\end{tabular}

\begin{abstract}
Due to the emergence of the new information and communication technologies (ICT), it is necessary that health providers develop new skills in the use of ICT. Physicians, nurses and other professionals interact with patients every day, and ICT, especially the internet, can enhance the communication processes among them, providing timely information to patients about disease prevention, health promotion, as well as treatment of diseases. The new ICT skills can enable greater interaction between health personnel and patients through email, SMS, chat, forums, websites, Facebook, Twitter and other media. ICT skills are: access to health science information, management of health information and knowledge, generation and dissemination of health knowledge. The development of the new ICT skills required that people are digital literate. ICT skills are essential for success in the Network Society.
\end{abstract}

Cómo citar el artículo

Choque Larrauri, R. (2011). Las nuevas competencias TIC en el personal de los servicios de salud. Revista de Comunicación y Salud, 1(2), pp. 47-60.

DOI: http://doi.org/10.35669/revistadecomunicacionysalud.2011.1(2).47-60

\section{La Sociedad Red}

La denominada Sociedad Red tuvo sus antecedentes en la década de 1960. Como tal, una serie de investigadores y científicos, entre los cuales podemos destacar a McLuhan (1964), Mead (1971), Masuda (1980) y Toffler (1981), entre otros, empezaron a hablar de un nuevo tipo de sociedad que se estaba empezando a forjar de una manera muy rápida y extendida, generando grandes transformaciones en diversos campos de la sociedad como las comunicaciones, la economía, la política, la educación, la salud pública, etc., y que respondía a la incursión de las nuevas TIC, principalmente la televisión y posteriormente internet. Al correr los años este nuevo tipo de sociedad se acuñó con el término de Sociedad Red por el holandés Jan van Dijk (1991), siendo Castells (2001) quien se encargó de contribuir a su desarrollo y promoción. 
En primer lugar conoceremos los aportes de Marshall McLuhan (1964), quien denominó el nuevo tipo de sociedad como la Sociedad Electrónica, asumiendo la máxima de que el mensaje es el medio. Para este profesor canadiense de Literatura toda tecnología significaba la extensión de nuestro cuerpo, nuestra mente y nuestro ser. En esta perspectiva señalaba que la ropa era una extensión de la piel; la casa era una extensión de los mecanismos de control de la temperatura del cuerpo; el estribo, la bicicleta y el automóvil eran extensiones del pie humano y la computadora una extensión de nuestro sistema nervioso central. Asimismo acuñó el término de "aldea global", que aludía a una situación en que todas las personas estaban interconectadas en el mundo. Como tal, McLuhan puso mucha atención en los cambios que los nuevos instrumentos generaban, insistiendo no sólo en su aplicación, sino en su efecto físico y especialmente cognitivo, ya que hay que darse "cuenta de que la adopción de nuevos instrumentos lleva aparejados grandes cambios en la forma ordinaria de hablar, actuar y pensar".

McLuhan asumía que la incursión de las nuevas TIC no solo producía cambios y transformaciones a nivel macro, sino que también se generaban cambios y transformaciones en las personas, es decir, en cada uno de los sentidos de las personas. Así, establecía que la interacción entre nuestros sentidos era perpetua. Sin embargo cuando los sentidos entraban en interacción con las nuevas tecnologías, éstas cambiaban en la forma de percepción a la que estaban acostumbradas.

Desde otra mirada, Margaret Mead (1971), una antropóloga cultural norteamericana muy reconocida, sostenía que estábamos en la Cultura Prefigurativa, donde por primera vez los seres humanos se habían congregado, en razón de las informaciones que los unos tenían acerca de los otros y de las reacciones que los unos provocaban en los otros, conformando una comunidad unida por el conocimiento. Esta situación es muy importante ya que antes fue imposible incluso siquiera conocer qué es lo que estaba pasando en los otros continentes y se desconocían las culturas que existían. Mead señaló también que un aspecto esencial y extraordinario del estado actual del hombre consistía en que nos aproximamos a una cultura mundial y a la posibilidad de convertirnos en ciudadanos totalmente conscientes del mundo. Este estado es una situación que nunca se presentó antes en la historia de la humanidad y que, por su naturaleza, nunca podría repetirse en estas condiciones.

Para especificar con mayor detalle este desarrollo de la humanidad, Margaret Mead (1971) señaló que en la historia de la humanidad existieron tres tipos de culturas: postfigurativa, cofigurativa y prefigurativa. Las distinciones que marcó entre tres tipos diferentes de cultura postfigurativa, en la que los niños aprenden primordialmente de sus mayores; cofigurativa, en la que tanto los niños como los adultos aprenden de sus pares, y prefigurativa, en la que los adultos también aprenden de los niños- son un reflejo del periodo en que vivimos. La cultura postfigurativa es aquella en que el cambio es tan lento e imperceptible que los abuelos, que alzan en sus brazos a los nietos recién nacidos, no pueden imaginar para estos un futuro distinto de sus propias vidas pasadas. La cultura cofigurativa es aquella en que el modelo prevaleciente para los miembros de la sociedad reside en la conducta de sus contemporáneos. La cultura prefigurativa es un nuevo estilo, porque en esta nueva cultura será el hijo, y no el padre ni los abuelos, quien representará el porvenir.

Como se ve, Margaret Mead con muchísimo tino nos hizo ver que estamos en un periodo 
donde no sabemos cómo serán los niños de los próximos años, cómo aprenderán, cómo funcionará su mente. Entonces nos queda a nosotros una tarea muy importante que es construir las condiciones necesarias en el entorno para que este niño, todavía desconocido, esté seguro y pueda crecer y descubrirse a sí mismo, a su comunidad y al mundo donde vive. Por tanto, conocer las competencias TIC, y a la vez difundirlas, resuelve en parte lo que Mead reclamaba.

Desde otro contexto, pero con la misma insistencia, Yoneji Masuda acuñó el término de Sociedad de la Información. Así, señaló que este es el periodo durante el cual hay una innovación en la tecnología de la información, donde el poder de la transformación de la sociedad lo constituye la cantidad y calidad de información que se genere. Con ello estableció que en la Sociedad Industrial el poder se constituía en la cantidad de materiales que se producían y ahora, en la Sociedad de la Información, el poder está delimitado por la cantidad de información que se produce en todos los campos del desarrollo. Bajo esta perspectiva, uno de los grandes aportes que dirigió Masuda en Japón fue el Plan para la Sociedad de la Información. En este plan definió con mucha claridad todas las transformaciones que ocurrirían en los diversos campos de la sociedad, como son la economía, la comunicación, la nueva forma de la administración pública, la mejora de la salud, la educación, la producción, etc.

Masuda (1980) tuvo mucho interés en el tema de la información. En este sentido realizó un análisis minucioso de cómo se concretiza la información desde los albores de la humanidad hasta la actualidad. La concretización de la información consiste en que la información que produce el hombre se materialice en algo palpable, visible y que quede perennemente como un medio de transmisión de la cultura de una generación a otra generación. Concretizar la información para Masuda fue un punto de quiebre trascendental en el desarrollo de la sociedad, que se mantiene hasta la actualidad y que, obviamente para la educación, es un aspecto clave porque en el proceso de aprendizaje la información es un elemento imprescindible.

En el proceso de concretización de la información, Masuda estableció cuatro periodos a los que llamó revoluciones. La primera revolución fue la del lenguaje, donde no existió la concretización de la información porque la transmisión de la información era de boca a boca, es decir la transmisión solo era de forma oral y por ende se perdía en el tiempo. La segunda revolución fue de la escritura donde, con la aparición de los números y las letras, se empezó a concretizar la información; de esta manera podemos encontrar una serie de informaciones expresadas en números y letras que incluso nos permiten conocer la evolución de la sociedad. La tercera revolución fue de la imprenta, donde se concretizó la información en una serie de obras y documentos que se imprimían a gran escala y con difusión masiva. La cuarta revolución es de la computadora, donde la información se concretiza de manera electrónica. 


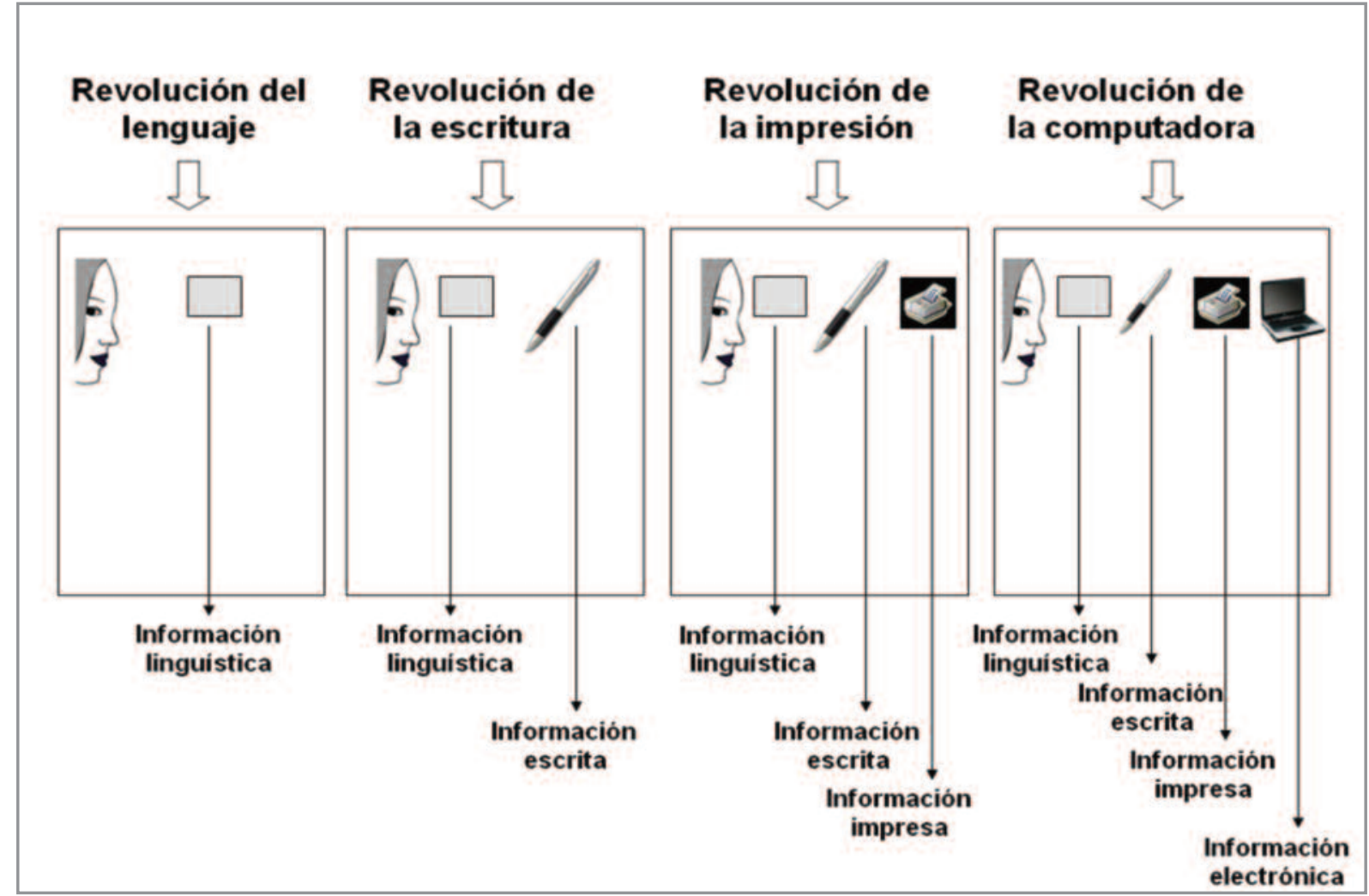

Fuente: Masuda, 1980.

Por tanto, el proceso de concretización de la información se ha dado hasta la actualidad en tres estadíos: primero la información fue escrita, segundo la información fue impresa y tercero la información es electrónica. Masuda tuvo un aporte muy importante en establecer el gran poder de la información en este nuevo tipo de sociedad.

Por otro lado, Alvin Toffler señaló el surgimiento de la Sociedad del Conocimiento, como el estado actual del desarrollo de la humanidad descrito como tres olas. La primera ola comenzó hacia el 8000 a.C. y dominó en solitario la tierra hasta los años 1650-1750 de nuestra era. A partir de este momento, la primera ola fue perdiendo ímpetu a medida que lo iba cobrando la segunda, la industrialización. La civilización industrial, producto de esta segunda ola, dominó a su vez el planeta hasta que también ella alcanzó su culminación. Este último punto de inflexión histórico llegó a los Estados Unidos durante la década iniciada alrededor de 1955, la década en que el número de empleados y trabajadores de servicios superó por primera vez al de obreros manuales. Fue esa la misma década que presenció la generalizada introducción de la computadora y muchas otras innovaciones de gran impacto. Bajo este contexto empezó a cobrar fuerza.

Cabe precisar que, para Toffler, una "ola" engloba una serie de consecuencias sociales, económicas, comunicacionales, educativas, psicológicas, etc., que se dan en cada una de las civilizaciones. Cada ola se clasifica de acuerdo a la tecnología que se usa para la producción, 
es decir, de acuerdo a una tecnosfera. La tecnosfera genera una forma de organización social, una determinada sociosfera. Así la sociosfera necesita de ciertos canales de comunicación que permitan entrelazar a la tecnosfera y la sociosfera; esto es, de una infosfera. En el desarrollo de la humanidad se han generado tres tipos de tecnosferas comunicativas que son la oral, la escrita y la electrónica. Siguiendo con este razonamiento, para Toffler cada una de las tecnosferas comunicativas condicionarán, por tanto, las infosferas y las sociosferas. Es decir, al cambiarse la tecnosfera también cambiará la sociosfera y la infosfera.

Esclareciendo la idea anterior, Toffler señala que, al alterar tan profundamente la infosfera, estamos destinados a transformar también nuestras propias mentes, la forma en que pensamos sobre nuestros problemas, la forma en que sintetizamos la información, la forma en que prevemos las consecuencias de nuestras propias acciones. Es posible que cambiemos el papel del analfabetismo en nuestras vidas. Puede, incluso, que alteremos nuestra propia química cerebral.

Para Toffler esta nueva sociedad, configurada como la tercera ola, trae consigo una serie de transformaciones y una forma de vida auténticamente nueva basada en fuentes de energía diversificadas y renovables, en métodos de producción diferentes, en nuevas familias no nucleares, en una nueva institución, en una nueva escuela y corporaciones radicalmente modificadas. La escuela que proponía salía de las cuatro paredes para ser una escuela abierta.

Tomando todo lo anterior encontramos con mucha claridad la concepción del surgimiento de un nuevo tipo de sociedad, pero de diferentes nombres: Sociedad Electrónica (McLuhan, 1968), Cultura Prefigurativa (Mead, 1971), Sociedad de la Información (Masuda, 1980) y Sociedad del Conocimiento (Toffler, 1981). Estas cuatro denominaciones de sociedad fueron bastante posicionadas e incluso en la actualidad se recurre a estos conceptos. Sobre esta base teórica aparecieron otros científicos sociales que también empezaron a trabajar en la conceptualización de este nuevo tipo de sociedad. Uno de los especialistas más reconocidos, y que continúa trabajando sobre este tema, es Manuel Castells.

Jan van Dijk (1991) acuñó el término de Sociedad Red y con ello entendió que un nuevo mundo está tomando forma en este fin de milenio. Se originó en la conciencia histórica de los años sesenta y mediados de los setenta, de tres procesos interdependientes: la revolución de la tecnología de la información; la crisis económica tanto del capitalismo como del estatismo y sus reestructuraciones subsiguientes; y el florecimiento de movimientos sociales y culturales, como el antiautoritarismo, la defensa de los derechos humanos, el feminismo y el ecologismo. La interacción de estos procesos y las reacciones que desencadenaron crearon una nueva estructura social dominante, la Sociedad Red; una nueva economía, la economía informacional/global; y una nueva cultura, la cultura de la virtualidad/real.

\section{Concepto de competencias TIC}

Es sumamente importante delimitar con mucha claridad el concepto de competencias, a fin de direccionar adecuadamente lo que significan y representan las competencias TIC. De acuerdo a Tobón (1999) las competencias son "procesos complejos que las personas ponen en acción- 
actuación-creación, para resolver problemas y realizar actividades de la vida cotidiana y del contexto laboral-profesional, aportando a la construcción y transformación de la realidad, para lo cual integran el saber ser (automotivación, iniciativa y trabajo colaborativo con otros), el saber conocer (observar, explicar, comprender y analizar) y el saber hacer (desempeño basado en procedimientos y estrategias), teniendo en cuenta los requerimientos específicos del entorno, las necesidades personales y los procesos de incertidumbre, con autonomía intelectual, conciencia crítica, creatividad y espíritu de reto, asumiendo las consecuencias de los actos y buscando el bienestar humano". Las competencias en tal perspectiva están constituidas por procesos subyacentes (cognitivo-afectivos), así como también por procesos públicos y demostrables en tanto implican elaborar algo de sí para los demás con rigurosidad.

Según Simone y Hersh (2004), el proyecto de la OCDE Definición y Selección de Competencias (DeSeCo) define la competencia como "la capacidad de responder a demandas complejas y llevar a cabo tareas diversas de forma adecuada". Supone una combinación de habilidades prácticas, conocimientos, motivación, valores éticos, actitudes, emociones y otros componentes sociales y de comportamiento que se movilizan conjuntamente para lograr una acción eficaz.

Las competencias son repertorios de comportamientos que algunas personas dominan mejor que otras, lo que les hace eficaces en una situación determinada. Así, al manejar las nuevas TIC, habrá personas que tengan mayor dominio en el uso del internet y otras personas estarán en proceso de aprendizaje. De esta manera es necesario precisar cuáles son esas competencias que las personas deben manejar adecuadamente en el campo de la salud pública. Una característica fundamental es que las competencias tecnológicas son universales, es decir no existen competencias del norte o del sur, pues el manejo de las computadoras y el internet tienen un lenguaje único, que es el hipertextual, en cualquier parte del mundo. Es como la matemática, que es igual en todo el mundo.

Uno de los fines principales de la educación es obviamente el desarrollo de competencias. La competencia desde el punto de vista educativo es la capacidad para aplicar los conocimientos, con eficiencia, eficacia y satisfacción, sobre algún aspecto de la realidad personal, social, natural o simbólica. Cada competencia es así entendida como la integración de tres tipos de saberes: conceptual (hacer), procedimental (saber hacer) y actitudinal (ser). 
Gráfico 2. Competencias TIC

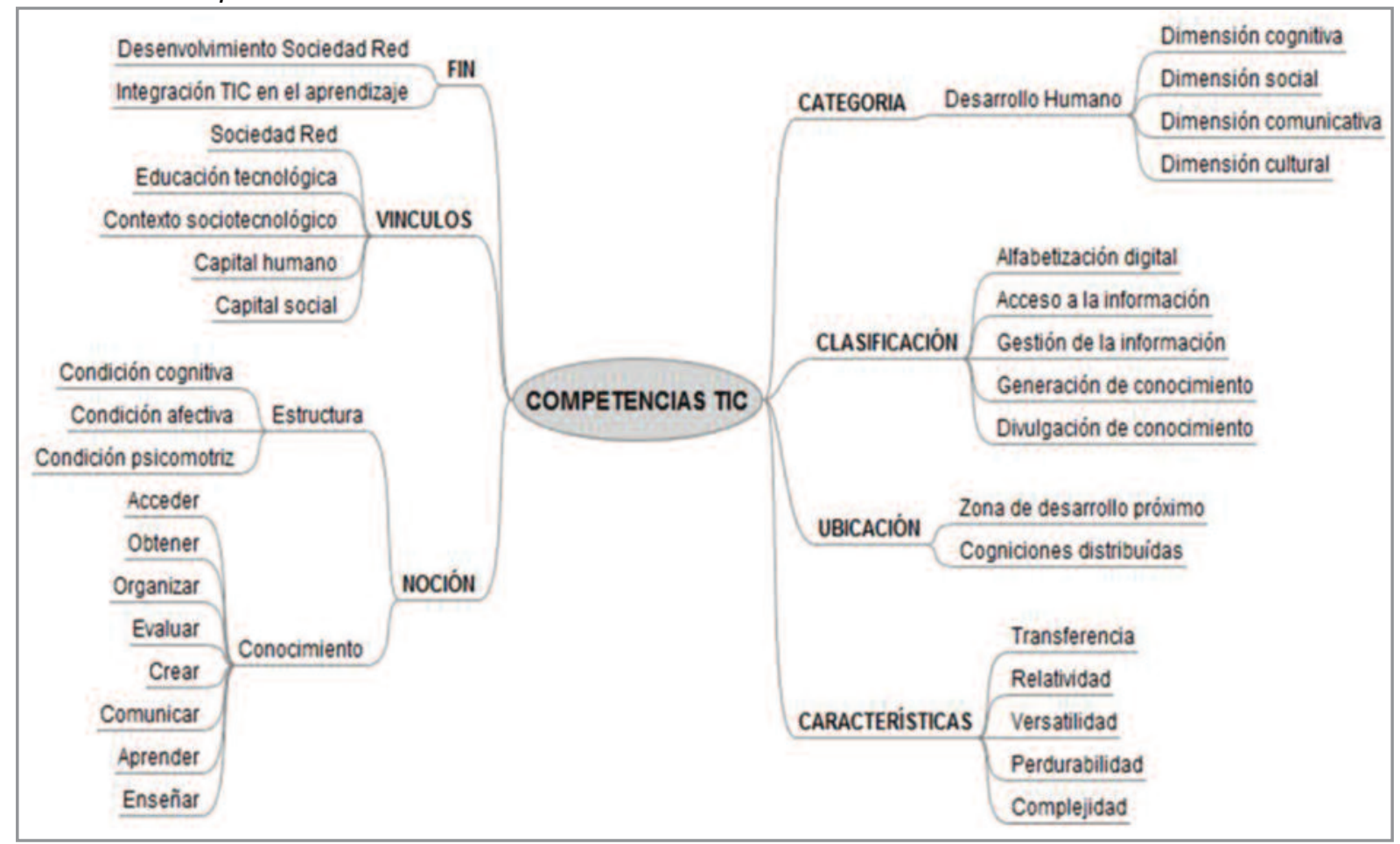

Fuente: elaboración propia.

En este gráfico se nos clarifica con mucha precisión todo lo que concierne a las competencias TIC, en los aspectos de la noción, los vínculos, la ubicación, la categoría, la clasificación, el fin y las características. Con base en esta organización conceptual, el concepto de competencias TIC que hemos desarrollado es el siguiente:

Las competencias TIC son las condiciones cognitivas, afectivas y psicomotrices que permiten a las personas utilizar las TIC para acceder, obtener, organizar, evaluar, crear y comunicar información, que les faciliten un pleno desenvolvimiento y desarrollo en la Sociedad Red.

Cabe precisar que la conceptualización que hemos dado acerca de competencias TIC está circunscrita al paradigma de la complejidad (Morin, 1990), por lo cual hemos considerado a la complejidad como epistemología de las competencias, implicando reconocer que es un enfoque inacabado y en constante construcción-deconstrucción-reconstrucción, requiriéndose continuamente del análisis crítico y la autorreflexión para comprenderlo y usarlo.

\section{Competencias TIC en el personal de salud}

El objetivo de este artículo es presentar cinco competencias que el personal de salud debe manejar adecuadamente para un desempeño eficiente en la Sociedad Red. Las cinco competencias TIC son las siguientes: uso eficiente de las TIC, acceso a la información y la comunicación, gestión de la información y el conocimiento, generación del conocimiento y difusión del conocimiento. 
En la Sociedad Red, donde actualmente nos encontramos desempeñándonos, el personal de salud requiere desarrollar una serie de conocimientos, habilidades y prácticas, que se configuran como las competencias TIC, a fin de que puedan brindar sus servicios a la población objetivo con mayor precisión, calidad y rapidez. De acuerdo con Lupiáñez (2010), internet ha emergido como un nuevo medio para la práctica médica, tal es así que la telemedicina y la teleasistencia son buenos ejemplos del uso de internet para proveer servicios sanitarios. Dentro de las nuevas prácticas del personal médico están, por ejemplo, el envío de partes médicos, seguimiento de consultas por vía electrónica (internet, mensajes a móvil), envío de SMS para recordar que el paciente debe tomar sus medicinas, pruebas médicas, visitas al centro de salud u hospital, consulta de la historia clínica, resultados de pruebas, etc. Asimismo, de acuerdo con Hawn (2009), la industria del cuidado de la salud desde los grandes hospitales viene implementando el uso con sus pacientes de nuevas herramientas como los blogs, los mensajes instantáneos, los vídeo-chat y el uso de las nuevas redes sociales, las cuales promueven una reingeniería en la interacción del médico y el paciente.

De acuerdo con Lupiáñez y otros (2010), un importante estudio realizado con la Asociación Médica de Barcelona (España) sobre la práctica médica en el uso de las TIC para su ejercicio profesional, señala que más de la mitad de los médicos informaron que utilizaron internet todos los días como parte de su práctica clínica. Para la mayoría de los médicos $(73,3 \%)$ internet es un recurso valioso que se utiliza para apoyar su práctica médica. Las principales fuentes de información a la que los médicos acceden son para conocer noticias médicas internacionales $(27,3 \%)$, revistas académicas internacionales $(18 \%)$ e información de su lugar de trabajo $(17,4 \%)$. El uso de internet para los médicos es principalmente para contactarse con otros profesionales de la salud y colegas $(89,9 \%)$, clarificar cuestiones acerca del diagnóstico, tratamiento u otros temas médicos $(74,7 \%)$ y contactarse por email con los pacientes $(22,5 \%)$. Otros datos muy importantes son que los médicos vienen realizando el envío de la prescripción de manera digital en el $4 \%$ de los casos y el envío de mensajes SMS para recordar que el paciente debe tomar sus medicinas en el $1,6 \%$.

En relación a los profesionales de enfermería, otro estudio realizado por Lupiáñez y otros (2011), señala que el $28,9 \%$ de enfermeras accede a internet como parte de su trabajo. Las otras actividades que realizan con mayor frecuencia son búsqueda de prácticas clínicas en bases de datos y páginas web $(24 \%)$, registros clínicos digitales de los pacientes $(18,1 \%)$, interactuar con los pacientes $(13,6 \%)$, búsquedas en revistas académicas nacionales $(12,1 \%)$, interactuar con otros profesionales de la salud en el país (8\%), publicar información $(2,4 \%)$ e interactuar con otros profesionales de la salud de otros países $(2,2 \%)$.

Estos datos nos muestran con mucha claridad que hay un cambio de paradigma en la interacción entre el profesional de la salud y los pacientes o usuarios que solicitan una atención o servicio de salud, obviamente a través del uso de las nuevas tecnologías de la información y la comunicación. Ahora ya no es novedad que a primera hora un usuario reciba un SMS de su médico indicándole que debe tomar su pastilla o también señalándole cuáles fueron los resultados de su último examen médico. En este nuevo panorama, entonces, cabe la necesidad de desarrollar las nuevas competencias TIC en el personal de salud, a fin de que puedan desempeñarse adecuadamente en la Sociedad Red. 
Las principales competencias que el personal que trabaja en el campo de la salud necesita desarrollar para desenvolverse adecuadamente en este nuevo contexto socio-tecnológico de la Sociedad Red son las siguientes:

\subsection{Uso eficiente de las TIC}

El uso eficiente de las TIC está referido a optimizar la prestación de servicios de salud a los ciudadanos, utilizando las TIC por el personal de salud, especialmente por los médicos, odontólogos, enfermeros, nutricionistas y demás profesionales de la salud. Con las TIC, especialmente a través de las páginas web, los foros, el email, el chat y los SMS, se pueden realizar un sinnúmero de actividades que acerquen en mayor medida al paciente o usuario del servicio de salud y se promueva la interacción entre el personal de salud y el paciente o usuario, de una manera rápida y sin tener límites en tiempos y espacios.

En esta competencia TIC para el personal de salud se proponen realizar las siguientes actividades:

- Manejo de la computadora

- Manejo del internet

- Administración de carpeta de datos

- Instalación de software

- Uso de correo electrónico, foro, chat, redes sociales, etc.

- Conocimiento del idioma universal científico que es el inglés

- Uso de la aplicación web Moodle para actividades de capacitación

- Uso de aulas virtuales

- Uso del sistema de comunicación interna de la organización

Por ejemplo, en relación con esta competencia, y de acuerdo a Lammintakanen y otros (2010), las enfermeras en un servicio de salud utilizan las TIC para la administración de la información del personal, la capacitación del personal, información administrativa y presupuestal, sistema clínico y archivo digital de los pacientes y sistema de comunicación interna a través de una intranet.

\subsection{Acceso a la información y conocimiento}

El acceso a la información y el conocimiento por el personal de salud es sumamente importante y es una de las principales actividades que realizan, puesto que la medicina es un campo de la ciencia donde cada día hay descubrimientos, nuevos fármacos, nuevos tratamientos, etc., por lo cual el personal está buscando permanentemente información en diferentes fuentes electrónicas. Sin embargo el acceso a la información y el conocimiento es una habilidad que se debe desarrollar a fin de acceder a información confiable y científica. Hay nuevas formas de acceder a nueva información, como por ejemplo las subscripciones a páginas web, a blogs, a diarios, a revistas especializadas, recibir alertas sobre nuevas publicaciones, etc. 
En esta competencia TIC para el personal de salud se propone realizar las siguientes actividades:

- Identificación de páginas web de salud

- Identificación de páginas web confiables

- Acceso a base de datos y revistas científicas en el campo de la salud

- Acceso a la base de datos PubMed

- Subscripción a revistas, periódicos, páginas web, foros, etc.

- Acceso a sitios web de organizaciones internacionales y nacionales de salud

\subsection{Gestión de la información y el conocimiento}

Un tema de bastante utilidad es la gestión de la información y el conocimiento en el campo de la salud. Hoy con el uso del internet se pueden tener las historias clínicas en línea e interconectadas, lo que permite a los profesionales de la salud acceder a toda la información necesaria para fines de diagnóstico, tratamiento, seguimiento y evaluación de los usuarios o pacientes de los servicios de salud. En tal sentido la gestión de la información es un aspecto fundamental que se tiene que promover en el personal de los servicios de salud a fin de que tengan las competencias necesarias para la creación de bases de datos y acceso a la información.

En esta competencia TIC para el personal de salud se propone realizar las siguientes actividades:

- Organización de la información en carpetas

- Organización de base de datos

- Clasificación de información científica e información en general

- Creación de historias clínicas en línea

- Elaboración de reportes de los pacientes y usuarios de los servicios de salud

- Digitalizar los resultados de los exámenes médicos, nutricionales, etc.

En muchos servicios de salud ya se viene implementando la e-prescripción, la cual ha tenido un impacto positivo pues permite acceder a toda la información de los pacientes y hacer el seguimiento de que los pacientes están tomando sus medicamentos. Por otro lado los médicos pueden acceder a la información de sus pacientes desde cualquier lugar y pueden interactuar con sus ellos (Goldman, Dubé y Lapane, 2010).

\subsection{Generación de conocimiento}

Generar conocimiento en el campo de la salud es una tarea que se desarrolla cotidianamente y que se realiza desde muchísimos años atrás. Sin embargo ahora tenemos las TIC que permiten que la generación del conocimiento sea compartida a nivel mundial sin ninguna frontera, por lo cual el personal de salud debe estar cada vez más preparado para poder difundir los nuevos conocimientos que generan producto de las investigaciones científicas que realizan. 
En esta competencia TIC para el personal de salud se propone realizar las siguientes actividades:

- Ejecución de investigaciones científicas en el campo de la salud pública

- Elaboración de artículos académicos y científicos

- Conocimiento de los requisitos mínimos para redactar artículos científicos

- Elaboración de reportes para publicación científica

\subsection{Difusión del conocimiento}

La difusión del conocimiento en el campo de la salud es un aspecto fundamental, ya sea para el propio personal de los servicios de salud, así como también para los usuarios del servicio de salud y para la comunidad en general. Para tal fin, con las TIC se puede lograr difundir (mediante páginas web, foros, SMS, etc.) mensajes a millones de personas a nivel mundial. Internet, como sabemos, es el medio más democrático en las naciones donde no se tiene ninguna restricción, con la cual se puede difundir información de manera rápida, óptima e incluso en los idiomas locales.

En esta competencia TIC para el personal de salud se propone realizar las siguientes actividades:

- Difusión de investigaciones y artículos científicos en revistas indexadas a nivel internacional

- Presentación de reportes de investigación en salud pública en eventos académicos

- Elaboración de revistas digitales científicas

- Elaboración de una página web

- Apertura de un foro de discusión sobre temas de salud

- Envío de SMS a trabajadores del servicio de salud y otros usuarios

- Utilización de las redes sociales para la difusión de información en salud

Las competencias que se han presentado son las principales que se deben promover en el personal de salud, sin embargo estas se irán incrementando y a la vez especializándose, pues las TIC también cada día van evolucionando y nos van presentando una serie de nuevas formas efectivas de comunicar salud, tanto al personal de los servicios de salud, como también a los ciudadanos en general.

\section{Conclusión}

En la actual Sociedad Red el personal de salud tiene un enorme reto en el aprovechamiento de los nuevos recursos tecnológicos que se nos presentan, a fin de que puedan realizar su labor de una manera más eficiente, efectiva y en el menor tiempo posible.

Ahora tenemos al alcance una serie de nuevas herramientas tecnológicas como son las páginas web sencillas y las páginas web interactivas, blogs, tweets, wikis, iPods, smartphones, las redes sociales como Facebook, entre otras, que enriquecen enormemente el proceso de la 
comunicación. Si antes la comunicación cara a cara o por teléfono era la convencional y la más efectiva, hoy la comunicación se da en un modelo en que una persona comunica a muchos, incluso a millones, por ejemplo utilizando Facebook. Visto así, el personal de salud tiene que desarrollar una serie de nuevas competencias TIC que le permitirán desenvolverse adecuadamente en este nuevo contexto sociotecnológico.

Para este fin es necesaria la ejecución de una serie de competencias como son el uso adecuado de las TIC en salud, el acceso a la información y el conocimiento en salud, la gestión de la información y conocimiento en salud, la generación de conocimiento en salud y la difusión del conocimiento en salud.

Es importante señalar también que las competencias TIC en el futuro se tienen que desarrollar en los pacientes o usuarios de los servicios de salud, a fin de que puedan hacer sus citas, revisar sus diagnósticos, revisar los resultados de sus exámenes, comunicarse con su médico, etc. Por tanto las competencias TIC alcanzan al personal de salud, a los administrativos que dirigen los servicios de salud y también a los pacientes.

\section{Referencias}

Castells, Manuel. (2001). La era de la información (3a ed.). Madrid: Alianza Editorial.

Goldman, Roberta; Dubé, Catherine y Lapane, Kate. (2010). Beyond the Basics: Refills by Electronic Prescribing. International Journal of Medical Informatics. $\mathrm{N}^{\circ} 79$, pp. 507-514. doi: 10.1016/j.jmedinf.2010.04.003

Hawn, Carleen. (2009). Take Two Aspirin and Tweet Me In The Morning: How Twitter, Facebook, and Other Social MediaAre Reshaping Health Care. Health Affairs. Vol. 28, n² 2. doi: 10.1377/hlthaff.28.2.361

Lammintakanen, Johanna; Saranto, Kaija y Kivinen, Tuula. (2010). Use of Electronic Information Systems in Nursing Managment. International Journal of Medical Informatics. $N^{\circ} 79$, pp. 324-331. doi: 10.1016/j.jjmedinf.2010.01.015

Lupiáñez, Francisco. (2010). Salud y Sociedad Red. Barcelona: Ariel, Editorial UOC y Generalitat de Catalunya.

Lupiáñez, Francisco; Hardey, Michael; Torrent, Joan y Ficapal, Pilar. (2010). The Integration of Information and Communication Technology into Medical Practice. International Journal of Medical Informatics. $\mathrm{N}^{\circ} 79$, pp. 478-491.

Lupiáñez, Francisco; Hardey, Michael; Torrent, Joan y Ficapal, Pilar. (2011). The Integration of Information and Communication Technology into Nursing. International Journal of Medical Informatics. $\mathrm{N}^{\circ} 80$, pp. 133-140.

Masuda, Yoneji. (1980). The Information Society as Post-Industrial Society. Washington D.C: 
World Future Society.

McLuhan, Marshall. (1968). El aula sin muros. En Carpenter, Edmun y McLuhan, Marshall. El aula sin muros. Investigación sobre técnicas de comunicación. Barcelona: Editorial de Cultura Popular.

McLuhan, Marshall. (1964). Understanding Media. The Extensions of Man. Londres: Routledge Classics.

Mead, Margaret. (1971). Cultura y compromiso: estudio sobre la ruptura generacional. Buenos Aires: Granica.

Morin, Edgar. (1990). Introducción al pensamiento complejo. Barcelona: Gedisa.

Simone, Dominique y Hersh, Laura. (2004). Definir y seleccionar las competencias fundamentales para la vida. México D.F.: Fondo de Cultura Económica.

Tobón, Sergio. (2004). Formación basada en competencias. Bogotá: ECOE Ediciones.

Toffler, Alvin. (1981). La tercera ola ( $7^{\circ}$ ed.). Barcelona: Plaza \& Janes.

Van Dijk, Jan. (1991). Aspects of New Media. De netwerkmaastchappij Bohn Staflen Van Loghum. The Netherlands: Houten. 